\title{
The meteor-shower complex of comet C/1917 F1 (Mellish)
}

\author{
L. Neslušan ${ }^{1}$ and M. Hajduková, jr. ${ }^{2}$
}

\author{
1 Astronomical Institute, Slovak Academy of Sciences, 05960 Tatranská Lomnica, Slovakia \\ e-mail: ne@ta3.sk \\ 2 Astronomical Institute, Slovak Academy of Sciences, Dúbravská cesta 9, 84504 Bratislava, Slovakia \\ e-mail: astromia@savba.sk
}

Received 8 January 2014 / Accepted 9 April 2014

\begin{abstract}
Aims. In our overall work, we attempt to predict some new meteor showers associated with as many as possible known periodic comets and to find the generic relationship of some already known showers with these comets. In this paper, we focus our attention on the meteor-shower complex of the long-period comet C/1917 F1 (Mellish), which is the known parent body of the December Monocerotids. Some other showers have also been suggested to be associated with this comet. We map its whole complex here.

Methods. For five perihelion passages of the parent comet in the past, we model associated theoretical streams, with each consisting of 10000 test particles and follow their dynamical evolution until the present. Subsequently, we analyze the orbital characteristics of the parts of found streams that approach the Earth's orbit.

Results. We confirm the generic relationship between the studied parent comet and December Monocerotids. The comet is probably also the parent body of the April $\rho$-Cygnids. The evolution of meteoroids to the orbits of April $\rho$-Cygnids is very long at about 20 millennia. If we follow even a longer evolutionary period, which is up to 50 millennia, then two diffuse showers with the radiant situated symmetrically to both the December Monocerotids and April $\rho$-Cygnids showers with respect to the apex of the Earth's motion occur. Our simulation does not confirm any relationship between C/1917 F1 and the November Orionids, although this shower was found in all three databases of observed orbits.
\end{abstract}

Key words. comets: individual: C/1917 F1 (Mellish) - meteorites, meteors, meteoroids

\section{Introduction}

Cometary nuclei are known to become active bodies, especially in the vicinity of the Sun. The gas flowing out of their surface drifts the smaller or larger dust particles, which then moves each in an orbit similar to the orbit of the parent comet. A swarm of all released particles forms the meteoroid stream, which usually fills the whole space in the vicinity of the entire parent's orbit after a period. The whole orbits of all such particles are situated in a space, which we refer to as a spatial "corridor". (In other words, the border of the corridor wraps around the orbits of the stream.)

Until the stream is not significantly perturbed, the orbit of the parent comet is situated inside the corridor at its center. If this orbit is situated relatively close to the orbit of the Earth at the same time, the particles also pass relatively close to the orbit of our planet, and some of them collide with its atmosphere. The effects of the interaction of a meteoroid and the Earth's atmosphere are called as a meteor, and the meteors originating in the given stream are associated with the corresponding meteor shower. The incoming directions of the meteors of a given shower are similar. This similarity is used to identify the members of the shower.

Sometimes, the perturbations of big planets can change the orbits of a part of stream in either a chaotic or systematic way. As a consequence, an alternative corridor or corridors of orbits can form. If more than a single corridor of a given stream passes through the Earth's orbit, we observe several meteor showers associated with the same parent body. The alternation of the initial orbital corridor can be revealed by modeling the stream and studying its dynamical evolution for a suitably long period. In the past, the meteoroid streams of several parent bodies were studied with help of various stream models (e.g., Williams \& Wu 1994; Wu \& Williams 1995; Brown \& Jones 1998; Asher 1999; Asher et al. 1999a,b; Neslušan 1999; Beech 2001; Asher \& Emel'yanenko 2002; Lyytinen \& Jenniskens 2003; Asher 2005; Vaubaillon et al. 2005a,b; Wiegert et al. 2005; Kaňuchová \& Neslušan 2007; Vaubaillon \& Jenniskens 2007; Asher 2008; Babadzhanov et al. 2008; Jenniskens \& Vaubaillon 2010; Vereš et al. 2011; Tomko \& Neslušan 2012; Babadzhanov et al. 2013; Jopek et al. 2013; Neslušan et al. 2013a,b; Sekhar \& Asher 2013, 2014a,b).

In this paper, we model and study the dynamical evolution of the meteoroid stream of the long-period comet C/1917 F1 (Mellish). The association of this comet to the Monocerotids as predicted by Porter (1952) and Hasegawa (1962), as well as suggested by Whipple (1954) on the basis of the first photographic catalogs, was later independently confirmed in several searches (McCrosky \& Posen 1961; Lindblad 1971; Kresáková 1974; Drummond 1981; Olson-Steel 1987; Ohtsuka 1989). The relationship was also studied by Lindblad and Olson-Steel (1990), who summarized the knowledge of the stream, as determined from various observations. They noticed, in several studies, that the Monocerotids are contaminated by members of another meteor shower, the $\xi$-Orionids (currently designated as November Orionids), which caused a discrepancy in the determinations of their radiants and orbits in various studies.

Hindley \& Houlden (1970) suggested another association of the comet Mellish to the 11 Canis Minorids. However, this shower was established only on the basis of five meteors. (It cannot be well identified with any meteor shower in the list by the IAU Meteor Data Center.) 
Kresáková (1974), who analyzed the Harvard photographic data, divided the meteors connected with the comet Mellish into two groups. This division was motivated by the asymmetrical distribution of their semi-major axes and supported by the distribution of their angular elements. The members of the longperiod group were identical to the Monocerotids and evidently associated with Comet Mellish. The short-period group, according to the author, could possibly have corresponded to the 11 Canis Minorids (in papers by Hindley \& Houlden 1970; and Kresáková 1974, number 11 is the catalog number of a star in the Canis Minor constellation, not the number of the shower in the IAU MDC list considered in Sect. 3.) or have been a symmetrical southern branch of the Geminid stream.

In a recent study of meteor showers connected to the comet Mellish, based on an analysis of the video meteor orbits from the SonotaCo database, Vereš et al. (2011) suggested the association of both December Monocerotids and November Orionids to this comet. Their common origin was supported by similar orbital characteristics, physical properties, and the narrow interval of the Southworth-Hawkins $(1963) D$-discriminant $\left(D_{\mathrm{SH}}<0.15\right)$ for most members of both streams with respect to the comet. However, the orbital evolution did not explain the inclination of November Orionids, which is about $10^{\circ}$ lower. The authors could not confirm the existence of the December Canis Minorids on the basis of the data analyzed.

As has been recently demonstrated in the cases of the comet 96P/Machholz and the asteroid $2003 \mathrm{EH} 1$, the modeling of a numerous theoretical meteoroid stream of the parent body and the following of its evolution can reveal a more complicated structure of the parts of stream that pass near the orbit of Earth and can cause the meteor showers (Neslušan et al. 2013a,b). In this paper, we perform an analogous modeling of the stream of comet $\mathrm{C} / 1917 \mathrm{~F} 1$. We expect that some further details in the structure of its stream could be revealed and the relationship with the known showers could be either confirmed or shown doubtful, or, maybe, a new relationship could appear.

As in the paper by Neslušan et al. (2013a), we use words "complex" and "stream" in the meaning of synonyms referring to the whole structure of meteoroids released from the parent body. The complex consists of several "filaments". If the particles of a filament hit the Earth, they can cause the meteor "shower" that corresponds to the filament.

\section{Modeling the stream}

To study the theoretical stream of comet C/1917 F1, we utilize the procedure suggested by Neslušan (1999), which was slightly modified and described in more detail by Tomko \& Neslušan (2012). Below, we recall the basic steps of the procedure and their application to the case of the parent comet under study.

In the first step, we integrate the orbit of the comet backward in time. The catalog orbit of C/1917 F1 was determined by Asklöf (1932). Unfortunately, the available astrometric observations of the comet enabled its orbit determination with precision that is not very high. The heliocentric ecliptic orbital elements $^{1}$ referred to the equinox J2000.0 are $q=0.190186 \mathrm{AU}$, $a=27.6473325 \mathrm{AU}, e=0.993121, \omega=121.3190^{\circ}, \Omega=$ $88.6683^{\circ}, i=32.6828^{\circ}$, and $T($ JDT $)=2421329.6751$ for epoch 2421333.5 . For the purpose of our modeling, we consider only this nominal (catalog) orbit.

\footnotetext{
1 Smithsonian Astrophys. Obs., 2008, http://ssd.jpl.nasa.gov/ sbdb.cgi
}

The end time of the comet-orbit integration is the moment of the comet perihelion passage, which is the closest to the end of a chosen period. The stream is modeled in this perihelion. Specifically, we create five models in the integration periods of $10,20,30,40$, and $50 \mathrm{kyr}$, approximately.

The evolution of the comet's orbit during the last 50 thousand years is shown in Fig. 1. The corresponding evolution of the minimum distance between this orbit and the Earth's orbit can be seen in Fig. 2, and the evolution of the position of orbital nodes with respect to the Earth's orbit is in Fig. 3. The current minimum distance is $\sim 0.1 \mathrm{AU}$ and decreases. This implies that the comet is a good candidate for the parent body of a meteor shower or, possibly, more showers.

When the perihelion in a chosen time is reached in the backward integration of comet orbit, the theoretical stream is modeled by assuming an ejection of 10000 test particles from the comet nucleus. The radius-vector of each particle is identical to the radius-vector of comet, and the particle velocity vector is calculated by adding a small, randomly oriented vector to the velocity vector of comet. The radius and velocity vectors of particle enable us to construct its orbit, which is similar to the orbit of the parent comet because the magnitude of the added velocity vector is small (one thousandth of comet perihelion velocity in the moment of stream modeling - that is, 103.5, 106.6, 104.9, 95.0 , and $69.6 \mathrm{~m} \mathrm{~s}^{-1}$ in our models).

After modeling the stream, we integrate the orbits of all particles from the moment of modeling until the present. We note that we use the integrator RA15 developed by Everhart (1985) within the MERCURY package (Chambers 1999) in all integrations. The gravitational perturbations of eight planets from Mercury to Neptune are considered.

When the integration of particle orbits is finished, we select the particles in the orbits that pass within 0.05 AU from the Earth's orbit. These particles are used to predict the corresponding meteor showers. If there are enough particles, we search for the predicted shower in the databases of actually observed showers. In this search, the photographic IAU MDC (Lindblad et al. 2003), radio-meteor (Hawkins 1963; Sekanina \& Southworth 1975; Lindblad 2003) and the SonotaCo video-meteor databases (SonotaCo 2009) are used. To identify a predicted shower to its potential counterpart in a given database, we use the break-point method (Neslušan et al. 1995, 2013c).

At the end of this section, we briefly discuss the reasons of our choice of the evolutionary periods from 10 to $50 \mathrm{kyr}$. The type of modeling, which we make, provides a good model of the stream with the particles distributed along the entire orbit of the parent body after at least a few tens of orbital revolutions. Since the orbital period of C/1917 F1 is 145 years, the modeled stream should evolve for a few millennia. The lower period of $10 \mathrm{kyr}$ is chosen with respect to this circumstance. Within a significantly shorter period than $10 \mathrm{kyr}$, only the December Monocerotids occur as a well-recognized shower. Since their relationship with the studied comet was already confirmed and this shower can also be studied in our models with the longer evolutionary periods, we do not create any models for the periods less than $10 \mathrm{kyr}$.

In addition, we focus our attention to the evolutionary periods that are longer than a few thousand years because of the following: Within the first few millennia after the stream formation, an occurrence of alternative corridors is improbable with respect to the relatively stable orbit of the parent comet and, hence, the expected stability of similar orbits of its meteoroids. A more complicated structure occurs after a longer evolution (and assumes that the comet orbit is not stable on a very long timescale, of course). It can be documented with the example of 

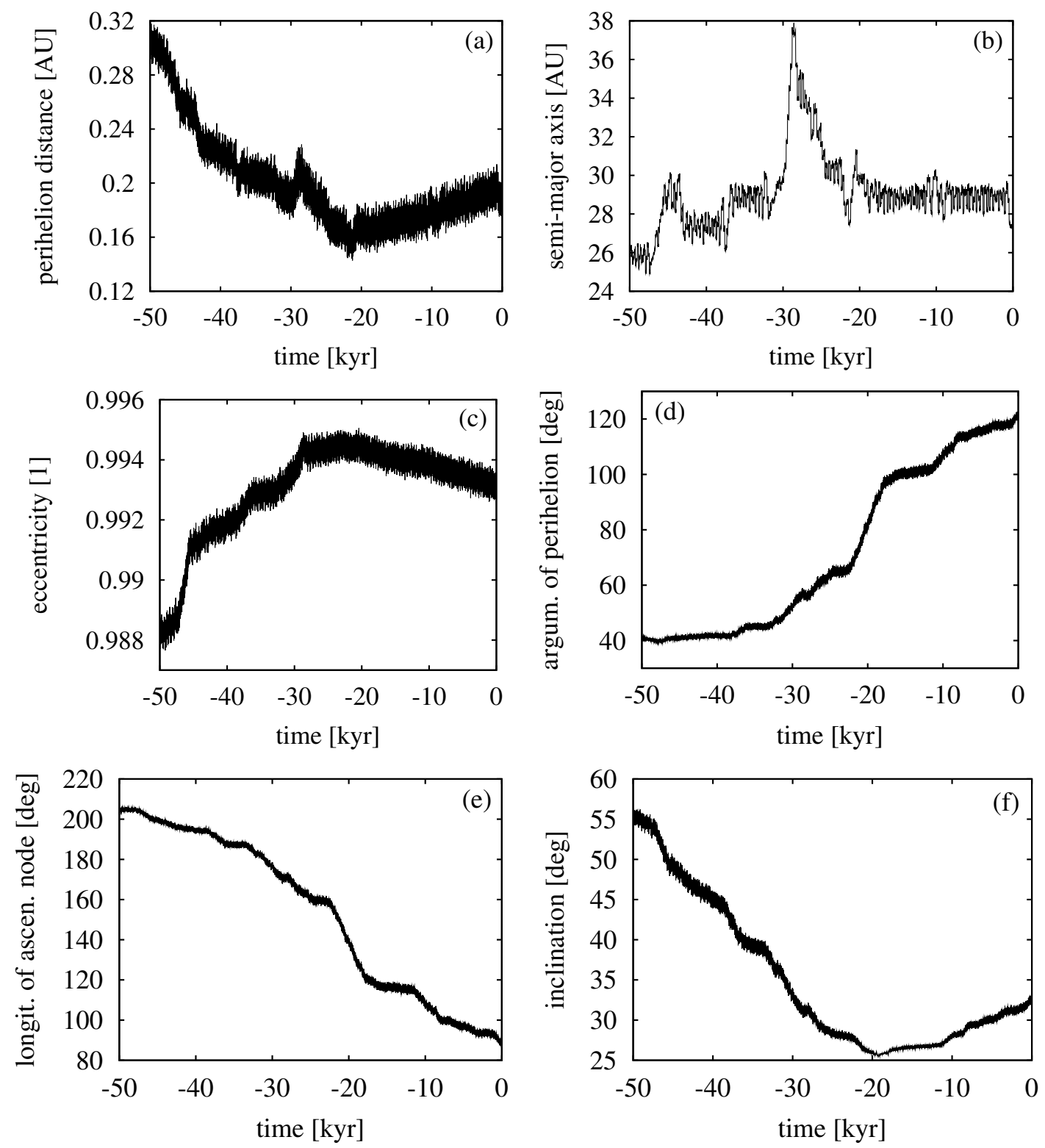

Fig. 1. Behavior of perihelion distance a), semi-major axis b), eccentricity $\mathbf{c}$ ), argument of perihelion d), longitude of ascending node e), and inclination to the ecliptic f) of the orbit of comet C/1917 F1 that has been reconstructed backward for 50000 years.

the stream simulated by Tomko $(2013,2014)$ for another longperiod comet $122 \mathrm{P} / \mathrm{de}$ Vico. In the models with the evolutionary period of 3718, 7435, and 18588 years, only 0,1 , and 3 orbits of particles are evolved, respectively, to approach the Earth's orbit at the present. A relatively numerous Earth-approaching strand (of 541 particles) occurred in the model with a period of 37175 years. And, a compact, well defined shower consisting of 1035 particles that approached the Earth's orbit yielded from Tomko's last model that assumed an evolutionary period of 55763 years. Simply, we can expect an occurrence of the Earthatmosphere observable shower from a given parent if there is enough time for the appropriate dynamical evolution of an associated stream into a complicated structure that has strands significantly different from the initial corridor of stream. Thus, we prefer the modeling that assumes the evolutionary periods last at least several ten thousand years.

According to Grün et al. (1984), the particles larger than $\sim 10^{-8} \mathrm{~kg}$ and moving in the Earth's vicinity and in lowinclination orbits are depleted on a timescale of $10^{4}$ years. Jenniskens (2006, p. 539) reported the estimate of an upper lifetime of millimeter-sized particles to be about $62 \mathrm{kyr}$. Wiegert et al. (2009) argued that the particles in a more inclined and larger orbits have a much longer collisional lifetime than claimed by Grün et al. In their simulations, they considered the evolutionary periods from 0.1 to $2.5 \mathrm{Myr}$, depending on the orbit of the parent. A less than $10 \%$ of the particles survived until the end of their simulations.

When choosing the value of an upper evolutionary period, we should remember the precision of C/1917 F1-orbit determination, which is not, unfortunately, very high. Integrating this orbit to the past, the probability of a significant discrepancy with reality increases. Hence, we do not consider a model with the evolutionary period exceeding $50 \mathrm{kyr}$, despite the result obtained by Wiegert et al. that the lifetime of meteoroid particles is longer than $10^{5} \mathrm{yr}$ (for some orbits longer than $10^{6} \mathrm{yr}$ ).

\section{The predicted showers}

The stream modeled for the time $10 \mathrm{kyr}(20,30,40$, and $50 \mathrm{kyr})$ ago approaches the Earth's orbit at the present in a single 
Table 1. The mean geophysical characteristics of the predicted meteor showers.

\begin{tabular}{|c|c|c|c|c|c|c|c|c|}
\hline Fil. & $t_{\mathrm{evol}} ;$ D.B. & $D_{\lim }$ & $\lambda_{\odot}$ & $\alpha$ & $\delta$ & $V_{\mathrm{g}}$ & $V_{\mathrm{h}}$ & $\gamma$ \\
\hline F1 & $20 \mathrm{kyr}$ & 0.19 & $39.706 \pm 3.801$ & $328.8 \pm 3.7$ & $50.0 \pm 2.2$ & $40.9 \pm 0.8$ & $41.4 \pm 0.1$ & 65.0 \\
\hline $\mathrm{F} 1$ & $30 \mathrm{kyr}$ & 0.19 & $37.427 \pm 3.056$ & $329.4 \pm 4.0$ & $49.2 \pm 2.1$ & $40.8 \pm 1.0$ & $41.5 \pm 0.1$ & 63.6 \\
\hline $\mathrm{F} 1$ & $40 \mathrm{kyr}$ & 0.21 & $37.609 \pm 4.084$ & $330.6 \pm 5.0$ & $48.3 \pm 2.7$ & $41.2 \pm 1.1$ & $41.5 \pm 0.1$ & 62.8 \\
\hline $\mathrm{F} 1$ & $50 \mathrm{kyr}$ & 0.28 & $37.769 \pm 5.045$ & $331.4 \pm 6.6$ & $48.0 \pm 3.1$ & $41.3 \pm 1.3$ & $41.5 \pm 0.1$ & 62.3 \\
\hline $\mathrm{F} 1$ & V & 0.18 & $37.302 \pm 3.407$ & $321.3 \pm 5.2$ & $45.3 \pm 3.4$ & $43.0 \pm 2.1$ & $40.6 \pm 0.9$ & 68.7 \\
\hline F2 & $40 \mathrm{kyr}$ & 0.17 & $154.628 \pm 7.089$ & $146.6 \pm 5.1$ & $31.8 \pm 2.8$ & $39.5 \pm 1.5$ & $42.1 \pm 0.2$ & 23.8 \\
\hline $\mathrm{F} 2$ & $50 \mathrm{kyr}$ & 0.14 & $158.604 \pm 4.984$ & $148.9 \pm 3.5$ & $29.3 \pm 2.0$ & $40.1 \pm 1.5$ & $41.9 \pm 0.3$ & 23.5 \\
\hline (F2 & V & 0.25 & $172.994 \pm 10.324$ & $152.6 \pm 7.3$ & $19.6 \pm 4.5$ & $40.5 \pm 4.0$ & $38.4 \pm 4.2$ & 26.5) \\
\hline F3 & $10 \mathrm{kyr}$ & 0.14 & $263.399 \pm 3.825$ & $103.9 \pm 2.9$ & $7.0 \pm 0.8$ & $41.3 \pm 1.2$ & $42.1 \pm 0.4$ & 154.0 \\
\hline F3 & $20 \mathrm{kyr}$ & 0.13 & $259.652 \pm 4.140$ & $100.6 \pm 3.1$ & $7.3 \pm 1.0$ & $41.5 \pm 1.4$ & $42.1 \pm 0.4$ & 153.7 \\
\hline F3 & $30 \mathrm{kyr}$ & 0.16 & $256.476 \pm 4.559$ & $97.7 \pm 3.2$ & $7.4 \pm 1.2$ & $41.6 \pm 1.4$ & $42.1 \pm 0.4$ & 153.6 \\
\hline F3 & $40 \mathrm{kyr}$ & 0.21 & $254.458 \pm 5.237$ & $96.5 \pm 3.6$ & $7.3 \pm 1.3$ & $42.0 \pm 1.4$ & $42.1 \pm 0.4$ & 152.8 \\
\hline F3 & $50 \mathrm{kyr}$ & 0.25 & $257.051 \pm 5.302$ & $97.8 \pm 3.8$ & $7.5 \pm 1.6$ & $41.3 \pm 1.5$ & $42.1 \pm 0.4$ & 154.0 \\
\hline$(\mathrm{F} 3$ & $\mathrm{F}$ & 0.10 & $260.064 \pm 2.529$ & $102.0 \pm 1.9$ & $7.8 \pm 0.7$ & $41.9 \pm 1.2$ & $41.8 \pm 0.6$ & 153.3) \\
\hline F3 & $\mathrm{F}$ & 0.25 & $256.443 \pm 6.404$ & $98.7 \pm 5.6$ & $10.3 \pm 3.6$ & $42.0 \pm 2.0$ & $41.9 \pm 1.4$ & 154.4 \\
\hline$(\mathrm{F} 3$ & V & 0.09 & $258.710 \pm 3.062$ & $100.8 \pm 2.2$ & $8.1 \pm 1.0$ & $41.4 \pm 1.0$ & $41.5 \pm 0.8$ & 153.3) \\
\hline F3 & V & 0.22 & $253.092 \pm 8.027$ & $96.1 \pm 6.4$ & $11.8 \pm 3.7$ & $41.7 \pm 2.0$ & $41.3 \pm 1.6$ & 154.4 \\
\hline F4 & $40 \mathrm{kyr}$ & 0.07 & $195.411 \pm 5.134$ & $70.8 \pm 5.3$ & $-33.1 \pm 0.1$ & $43.4 \pm 0.2$ & $41.3 \pm 0.0$ & 113.6 \\
\hline F4 & $50 \mathrm{kyr}$ & 0.14 & $186.915 \pm 6.152$ & $63.2 \pm 4.5$ & $-33.2 \pm 0.7$ & $43.5 \pm 0.3$ & $41.4 \pm 0.1$ & 115.5 \\
\hline (F4 & V & 0.23 & $202.680 \pm 3.385$ & $72.7 \pm 5.0$ & $-29.5 \pm 2.7$ & $42.6 \pm 2.3$ & $40.7 \pm 1.1$ & $117.2)$ \\
\hline
\end{tabular}

Notes. $t_{\text {evol }}$ - period of the following of orbital evolution (the theoretical stream was modeled before this time); D.B. - database from which the observed counterpart of the filament is selected (F - photographic, V - video-meteor); $D_{\text {lim }}$ - the threshold value of the Southworth-Hawkins $D$-discriminant to separate the meteors of the filament or observed shower; $\alpha_{\mathrm{g}}$ and $\delta_{\mathrm{g}}$ - equatorial coordinates of geocentric radiant; $V_{\mathrm{g}}$ and $V_{\mathrm{h}}-$ geocentric and heliocentric velocity; and $\gamma$-angular distance of the mean radiant from the Sun in time corresponding to the mean longitude. Time $t_{\text {evol }}$ is given in kilo-years; the angular quantities are given in degrees and velocities in $\mathrm{km} \mathrm{s}^{-1}$.

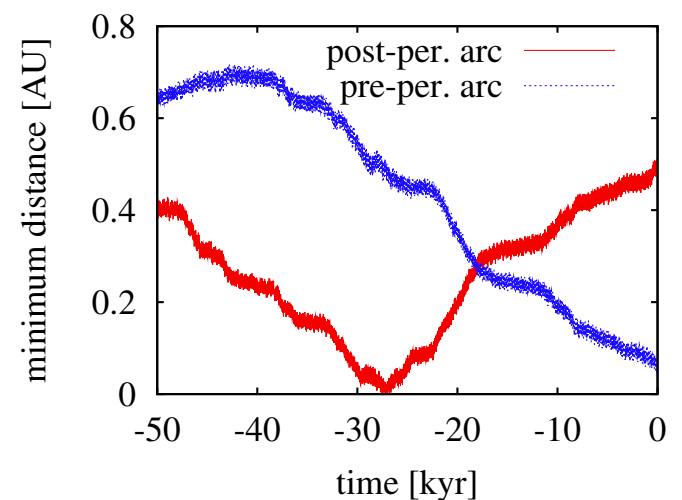

Fig. 2. Evolution of the minimum distance between the orbital arcs of comet C1917 F1 and the Earth's orbit from a time 50000 years before the present to the present. The minimum distance of the post-perihelion (pre-perihelion) arc is shown with the solid red (dashed blue) curve.

filament $(2,2,4$, and 5 filaments, respectively). The radiant areas of the individual filaments in the created models are shown in Fig. 4. In our further discussion, we omit the fifth filament with the most-right radiant area in Fig. 4e, since it occurs only in the 50-kyr model and consists of only few particles.

The position of the radiant area of each filament is used to divide the part of the stream that approaches the Earth's orbit into the individual filaments. Specifically, the filaments from F1 to F4 (Fig. 4e) are defined by

F1: $\alpha_{g}<10^{\circ}$ or $\alpha_{g}>300^{\circ}$;

F2: $130^{\circ}<\alpha_{g}<160^{\circ}$ and $20^{\circ}<\delta_{g}<40^{\circ}$;

F3: $80^{\circ}<\alpha_{g}<120^{\circ}$;

F4: $40^{\circ}<\alpha_{g}<80^{\circ}$ and $-40^{\circ}<\delta_{g}<-20^{\circ}$,

where $\alpha_{g}$ and $\delta_{g}$ are equatorial coordinates of geocentric radiant. The radiant areas of filaments F1 and F2 (F3 and F4) are situated northward (southward) from the ecliptic. The obtained mean

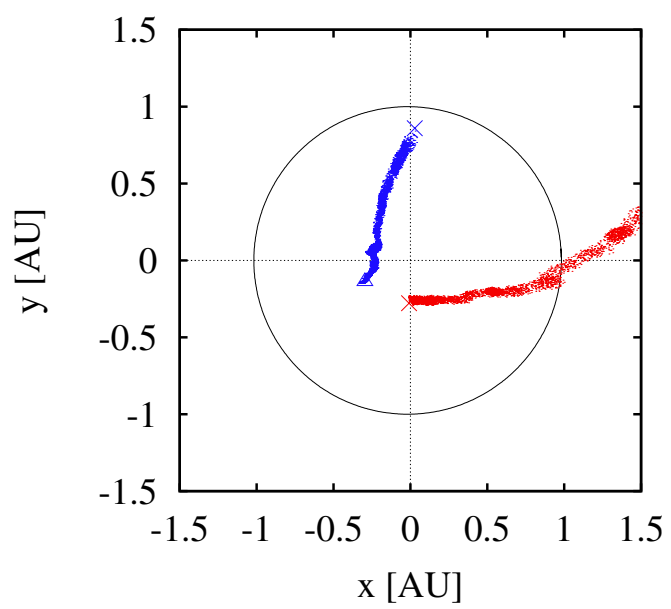

Fig. 3. Evolution of the orbital node position of comet C/1917 F1 during the last 50 thousand years. The red (blue) curve shows the shift in the descending (ascending) node that corresponds to the radiant northward (southward) of the ecliptic. The crosses indicate the starting positions (at the present) and the full triangles are the final position of the nodes in the considered period (only a part of the path of the descending node in a vicinity of the Earth's orbit is shown). The orbit of the Earth is shown with the circle.

geophysical characteristics of all four filaments in the models, where they exist, are given in Table 1 and the mean orbital elements in Table 2 .

The evolution of the distribution of orbital elements for the stream modeled at a time 40000 years before the present is shown in Fig. 5. Two clearly separated peaks evolve in the distributions of perihelion distance (Fig. 5a), eccentricity (5c), argument of perihelion (5d), and inclination (5f). In the distribution of longitude of ascending node (5e), the small third peak occurs after half of the studied evolutionary period. The high 

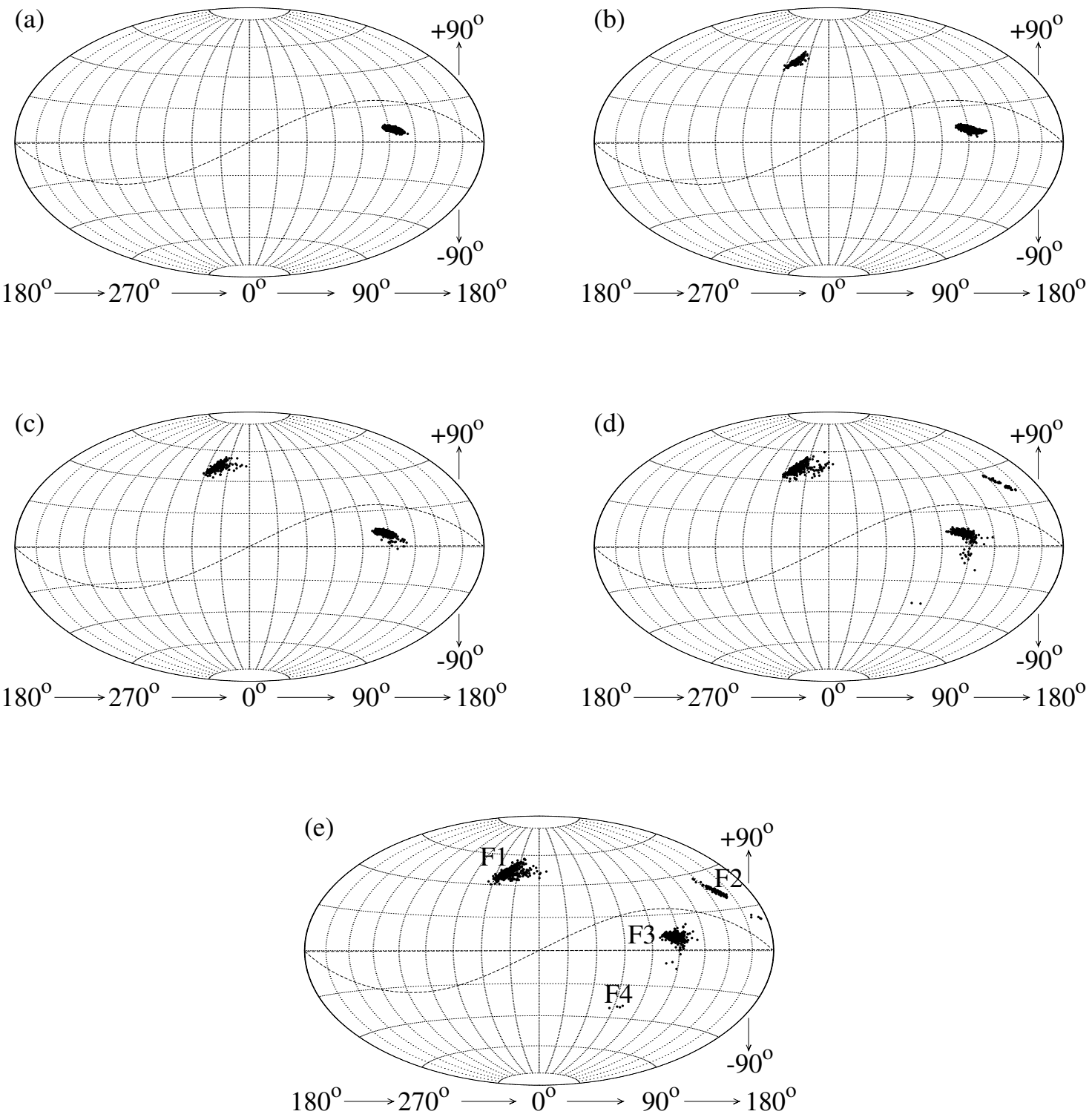

Fig. 4. Positions of radiants for the particles representing the modeled stream of comet C/1917 F1, which currently move in orbits, along which they can approach the Earth's orbit within $0.05 \mathrm{AU}$. The positions for the streams modeled in the perihelia of the comet passed at times of 10, 20, 30, 40, and $50 \mathrm{kyr}$ before the present are shown in the plots a), b), c), d), and e), respectively. The stream can be split up to four filaments with four distinct radiant areas. The enumeration of the filaments is given in plot e) with the complete set of filaments (F1 to F4). The radiants are shown in the Hammer projection of the equatorial coordinate frame. The sinusoid-like curve illustrates the ecliptic.

initial peak in the distribution of the semi-major axis (1b) slowly becomes lower and wider. The semi-major axis of part of the particle orbits increases during about a half of the period. At the end of evolution (i.e., in the present), this orbital element ranges from about 14 to $53 \mathrm{AU}$, whereby, no value in this interval is considerably preferred.

The specific features of the individual filaments in the considered models are described in the following subsections.

\subsection{December Monocerotids}

According to our modeling, the most numerous shower originating in the comet nucleus of $\mathrm{C} / 1917 \mathrm{~F} 1$ should be the one that corresponds to the theoretical filament F3. The meteoroids of this filament approach to the Earth's orbit relatively soon after their ejection from the nucleus. We identify this filament to the December Monocerotids (No. 19 in the IAU MDC list of the established showers ${ }^{2}$ ). The December Monocerotids were separated from all three used databases (Tables 1 and 2, filament F3, databases F, V, and R).

As stated in Sect. 2, we select the meteors of a given shower from the database using the "break-point method" (Neslušan et al. 1995, 2013c). Here, the predicted mean orbit of a given filament is considered as the initial orbit entering the iteration, which is done to select the meteors of a given shower from the database. For a given threshold value of the SouthworthHawkins (1963) $D$-discriminant, $D_{\text {lim. }}$, all meteors in the orbits with $D \leq D_{\text {lim. }}$ are selected, where $D$ is the value of the discriminant between the orbit of an individual meteor and an actual mean orbit of the shower; that is, the mean orbit calculated from all shower meteors selected at the previous iteration step. The

\footnotetext{
2 http://www.ta3.sk/IAUC22DB/MDC2007/Roje/roje_lista. php?corobic_roje=1\&sort_roje $=0$
} 
Table 2. Mean orbital characteristics with the dispersion (characterized by standard deviation) of the predicted meteor showers associated with the parent body considered.

\begin{tabular}{|c|c|c|c|c|c|c|c|c|c|}
\hline Fil. & $t_{\mathrm{evol}} ;$ D.B. & $D_{\lim }$ & $q$ & $a$ & $e$ & $\omega$ & $\Omega$ & $i$ & $N_{\text {sel. }}$ \\
\hline F1 & $20 \mathrm{kyr}$ & 0.19 & $0.819 \pm 0.028$ & $17.737 \pm 2.219$ & $0.953 \pm 0.006$ & $127.9 \pm 2.4$ & $39.7 \pm 3.7$ & $66.1 \pm 2.1$ & 176 \\
\hline F1 & $30 \mathrm{kyr}$ & 0.19 & $0.805 \pm 0.031$ & $21.329 \pm 3.422$ & $961 \pm 0.006$ & $125.7 \pm 3.0$ & $37.5 \pm 3.0$ & $65.3 \pm 2.4$ & 290 \\
\hline F1 & $40 \mathrm{kyr}$ & 0.21 & $0.790 \pm 0.035$ & $23.776 \pm 5.354$ & $.965 \pm 0.006$ & $123.4 \pm 3.8$ & $37.7 \pm 4.0$ & $66.0 \pm 2.8$ & 289 \\
\hline F1 & $50 \mathrm{kyr}$ & 28 & $0.779 \pm 0.045$ & $24.739 \pm 6.646$ & $967 \pm 0.007$ & $122.4 \pm 5.1$ & $37.8 \pm 5.0$ & $6.2 \pm 3.3$ & 406 \\
\hline $\mathrm{F} 1$ & $\mathrm{~V}$ & 18 & $0.841 \pm 0.029$ & $9.474 \pm 9.261$ & $894 \pm 0.070$ & $130.7 \pm 4.8$ & $37.3 \pm 3.4$ & $72.6 \pm 4.7$ & 22 \\
\hline $\mathrm{F} 1$ & $\mathrm{R}$ & 0.20 & $0.768 \pm 0.041$ & $23.569 \pm 37.297$ & $0.922 \pm 0.051$ & $120.5 \pm 5.2$ & $47.8 \pm 2.8$ & $67.2 \pm 5.8$ & 14 \\
\hline $\bar{F} 2$ & $40 \mathrm{kyr}$ & 0.17 & $0.291 \pm 0.044$ & $172.693 \pm 56.760$ & $0.998 \pm 0.000$ & $67.4 \pm 6.3$ & $152.5 \pm 8.0$ & $30.6 \pm 1.7$ & 35 \\
\hline $\mathrm{F} 2$ & $50 \mathrm{kyr}$ & 0.14 & $0.253 \pm 0.032$ & $75.925 \pm 23.856$ & $0.996 \pm 0.001$ & $60.9 \pm 4.4$ & $157.8 \pm 5.5$ & 1.7 & 89 \\
\hline$(\mathrm{F} 2$ & V & 0.25 & $0.115 \pm 0.051$ & $8.059 \pm 9.500$ & $0.969 \pm 0.036$ & $35.6 \pm 10.1$ & $173.0 \pm 10.3$ & $24.0 \pm 3.9)$ & 13 \\
\hline (F2 & $\mathrm{R}$ & 0.18 & $0.385 \pm 0.047$ & $2.259 \pm 0.826$ & $0.810 \pm 0.067$ & $66.8 \pm 6.8$ & $148.3 \pm 6.5$ & $33.7 \pm 3.3)$ & 10 \\
\hline$(\mathrm{F} 2$ & $\mathrm{R}$ & 0.17 & $0.222 \pm 0.051$ & $1.942 \pm 0.957$ & $0.865 \pm 0.060$ & $46.2 \pm 7.2$ & $178.2 \pm 6.8$ & $29.9 \pm 3.6)$ & 13 \\
\hline F3 & $10 \mathrm{kyr}$ & 0.14 & $0.213 \pm 0.020$ & $28.245 \pm 6.748$ & $0.992 \pm 0.002$ & $124.5 \pm 3.6$ & $83.9 \pm 5.0$ & $34.2 \pm 2.0$ & 1626 \\
\hline F3 & $20 \mathrm{kyr}$ & 0.13 & $0.206 \pm 0.024$ & $36.197 \pm 8.489$ & $0.994 \pm 0.002$ & $126.0 \pm 3.2$ & $79.7 \pm 4.7$ & $35.5 \pm 2.1$ & 898 \\
\hline F3 & $30 \mathrm{kyr}$ & 0.16 & $0.204 \pm 0.028$ & $37.793 \pm 7.138$ & $0.994 \pm 0.002$ & $126.4 \pm 4.0$ & $76.4 \pm 5.2$ & $36.2 \pm 2.6$ & 533 \\
\hline F3 & $40 \mathrm{kyr}$ & 0.21 & $0.197 \pm 0.030$ & $35.578 \pm 7.143$ & $0.994 \pm 0.001$ & $127.4 \pm 4.5$ & $74.5 \pm 5.7$ & $37.9 \pm 3.4$ & 531 \\
\hline F3 & $50 \mathrm{kyr}$ & 0.25 & $0.210 \pm 0.031$ & $34.549 \pm 6.890$ & $94 \pm 0.001$ & $125.2 \pm 4.6$ & $77.3 \pm 6.0$ & $34.9 \pm 3.8$ & 479 \\
\hline (F3 & $\mathrm{F}$ & 0.10 & $0.189 \pm 0.017$ & $53.035 \pm 67.069$ & $.990 \pm 0.012$ & $128.7 \pm 2.1$ & $80.1 \pm 2.5$ & $35.9 \pm 2.1$ & 14) \\
\hline F3 & $\mathrm{F}$ & 0.22 & $0.168 \pm 0.040$ & $100.343 \pm 210.941$ & $0.990 \pm 0.023$ & $132.1 \pm 6.1$ & $76.4 \pm 6.4$ & $31.7 \pm 5.7$ & 23 \\
\hline (F3 & V & 0.09 & $0.187 \pm 0.018$ & $15.571 \pm 20.716$ & $0.983 \pm 0.014$ & $129.3 \pm 2.7$ & $78.7 \pm 3.1$ & $35.3 \pm 1.9$ & 388) \\
\hline F3 & V & 0.22 & $0.153 \pm 0.045$ & $14.792 \pm 23.138$ & $0.984 \pm 0.021$ & $135.1 \pm 7.5$ & $73.1 \pm 8.0$ & $29.6 \pm 6.0$ & 1268 \\
\hline (F3 & $\mathrm{R}$ & 0.11 & $0.183 \pm 0.016$ & $8.749 \pm 4.346$ & $0.969 \pm 0.033$ & $131.1 \pm 2.4$ & $77.3 \pm 3.0$ & $35.4 \pm 2.7$ & 11) \\
\hline F3 & $\mathrm{R}$ & 0.16 & $0.146 \pm 0.045$ & $31.823 \pm 117.770$ & $0.976 \pm 0.036$ & $136.6 \pm 6.8$ & $72.2 \pm 6.5$ & $23.8 \pm 3.5$ & 62 \\
\hline F4 & $40 \mathrm{kyr}$ & 0.07 & $0.821 \pm 0.037$ & $12.681 \pm 1.000$ & $0.935 \pm 0.008$ & $53.9 \pm 2.9$ & $15.3 \pm 5.0$ & $71.8 \pm 0.7$ & 2 \\
\hline F4 & $50 \mathrm{kyr}$ & 0.14 & $0.753 \pm 0.017$ & $14.439 \pm 0.802$ & $0.948 \pm 0.003$ & $56.1 \pm 1.4$ & $7.2 \pm 6.2$ & $71.6 \pm 0.7$ & 4 \\
\hline (F4 & V & 0.23 & $0.746 \pm 0.034$ & $20.716 \pm 35.115$ & $0.893 \pm 0.079$ & $62.1 \pm 4.1$ & $22.7 \pm 3.4$ & $70.5 \pm 6.5$ & 7) \\
\hline
\end{tabular}

Notes. $t_{\mathrm{evol}}$ and $D_{\mathrm{lim}}-$ as in Table. 1; D.B. - database from which the observed counterpart of the filament is selected (F - photographic; V - videometeor; $\mathrm{R}$ - radio-meteor database); $q$ - perihelion distance; $a$ - semi-major axis; $e$ - eccentricity; $\omega$ - argument of perihelion; $\Omega$ - longitude of ascending node; $i$ - inclination to the ecliptic; and $N_{\text {sel. }}-$ the number of particles or real meteors selected from the modeled set (of 10000 particles) or from given database. Quantities $q$ and $a$ are given in astronomical units and angular elements in degrees.

iteration is finished when the difference, again measured with the help of $D$, of two successive mean orbits is $D<10^{-6}$. The break-point method assumes that the iteration for a series of $D_{\mathrm{lim}}$. values, typically ranging from 0.01 to 1.0 , repeats and that the dependence of the number of selected shower meteors, $N$, on the value of threshold $D_{\text {lim. }}$ is constructed.

The dependence of $N$ on $D_{\text {lim. }}$ for the photographic IAU MDC database is shown in Fig. 6, when the predicted mean orbit of filament F3 is considered as the initial orbit to the iteration. As seen, the first meteors of the shower corresponding to this filament are selected for $D_{\text {lim. }}=0.04$. With the increasing value of $D_{\text {lim. }}$, a larger and larger number of shower meteors is naturally selected. The selection reaches the first saturation stage for $D_{\text {lim. }}=0.10$ (marked by a left-hand side arrow in Fig. 6) when all meteors of the first shower's dense structure are selected. The point of which $N$ levels off represent the "break point" in the dependence.

Normally, there is only a single break point and a smooth, compact structure of shower. The real December Monocerotids appear to be exceptional in this sense because the iteration procedure first reveals a subfilament with the mean orbit being just coincident with the predicted theoretical mean orbit (the mean orbit of filament 3 . When we further increase $D_{\text {lim. }}$, the plateau in the $N=N\left(D_{\text {lim. }}\right)$ dependence ends with another steep increase of $N$, which starts at $D_{\text {lim. }}=0.21$. For $D_{\text {lim. }}>0.21$, the meteors belonging to the main structure of the shower are selected by the iteration procedure. The procedure changes the calculated mean orbit toward the mean orbit of the shower as a whole. In $D_{\text {lim. }}=0.22$ (marked with the right-hand side arrow in Fig. 6), there is another break point, which enables the selection of the main dense part of the shower.
If we further increase $D_{\text {lim. }}$, the meteors of a dispersed "tail" (in the orbital phase space) are selected. This tail used to not be very numerous. Because of this circumstance, an infiltration of the shower with the neighboring (in the phase space) meteors of sporadic background is relatively large and, hence, the method recommends that we consider only the meteors of the dense core to calculate the mean characteristic of the selected shower. (The method of the break point enables us to determine an optimal value of $D_{\text {lim. }}$ for selection of the well-defined members of the shower. If we, for example, selected the shower meteors from the database by considering the fixed value $D_{\text {lim. }}=0.20$, which has been often used, only the substructure of the shower in the database would be selected. In contrast, if we did the selection by considering the fixed value of $D_{\text {lim. }}=0.3$, for example, the shower would be contaminated with a relatively large number of the meteors, which do not belong to it.)

The mean data characterizing the shower 19 December Monocerotids in the used databases are also given in Tables 1 and 2 (parts of filament F3). The characteristics for the substructure consisting of meteors selected with $D_{\mathrm{lim}}=0.10$ in the photographical data and with $D_{\text {lim }}=0.09$ and 0.11 in the video and radio data, respectively, are given in parentheses. The whole shower was selected with $D_{\text {lim. }}=0.22,0.22$, and 0.16 in the photographic, video, and radio data, respectively.

In the phase space of orbital elements, another established shower, 250 November Orionids, occurs to be in the vicinity of 19 December Monocerotids. We note that the November Orionids are not related to comet C/1917 F1, according to our analysis. Although it is possible to select the members of the diffuse shower with the mean characteristics that corresponds to the November Orionids in the photographic and video databases, 

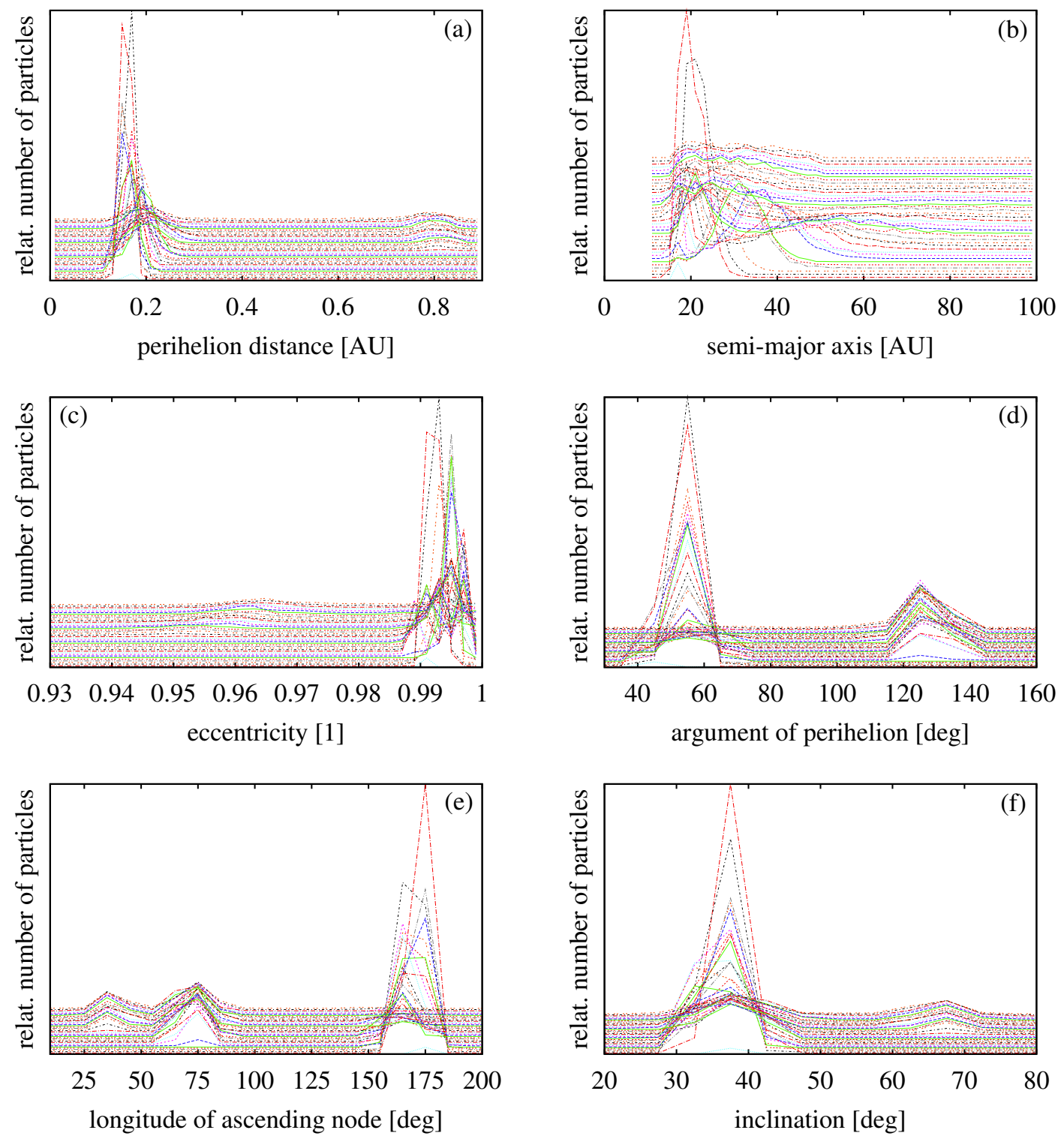

Fig. 5. Evolution of the distribution of perihelion distance a), semi-major axis b), eccentricity c), argument of perihelion d), longitude of ascending node e), and inclination to the ecliptic f) for the part of the C/1917 F1 stream modeled at a time 40000 years before the present. The bottom curve in each plot shows the initial distribution of a given element. Each successive curve, which is entirely shifted vertically about a small displacement above the previous one, shows the distribution after another 1000 years. The top curve shows the distribution at the present.

no such shower is separated in any of our models. Filament 3, which is closest to the November Orionids in the orbital phase space is smooth and not contain any accumulation of particles in the orbits in its border, similar to the real shower No. 250. Of course, the theoretical counterpart could be less numerous than the real shower and, thus, a corresponding break point could not be apparent. To exclude this possibility, we improve the iteration procedure to converge to two mean orbits. If the D-discriminant that compares the orbit of a particle and a given mean orbit is smaller than $D_{\text {lim. }}$ for both considered mean orbits, the particle is associated only to the more similar (with lower $D$ ) mean orbit. Even when using this improved procedure, no convergence to the mean orbit of real November Orionids occurs in the theoretical data.

One can speculate that the November Orionids could still be related to $\mathrm{C} / 1917 \mathrm{~F} 1$, whereby the members of this stream come to their orbits due to the non-gravitational effects, which we do not consider in our modeling. We know that the magnitude of the action of non-gravitational effects as a radiation or solar-wind pressure depends on the size of particles. In addition, the action of the effects on the particles of a different age, even if they are the same size, results in a different change of their initial orbit. Therefore, we would expect a smooth transition of orbits from those, which are typical for the parent comet and December Monocerotids, to the November Orionids, if the nongravitational effects were the agent that modifies the orbits of the $\mathrm{C} / 1917 \mathrm{~F} 1$ stream to become the November Orionids. This is, however, not the case. Constructing the common plot of, e.g., the distribution of right ascension of radiants for both showers, we find that there is a deep minimum between the peaks in the distribution, which correspond to the December Monocerotids and November Orionids. If the common plot is constructed for the radiant declination distribution using the photographical data, the peaks corresponding to both showers are, so far, clearly separated each from other. There is no meteor belonging to the first or second shower with the declination of radiant between $10^{\circ}$ and $14^{\circ}$. 


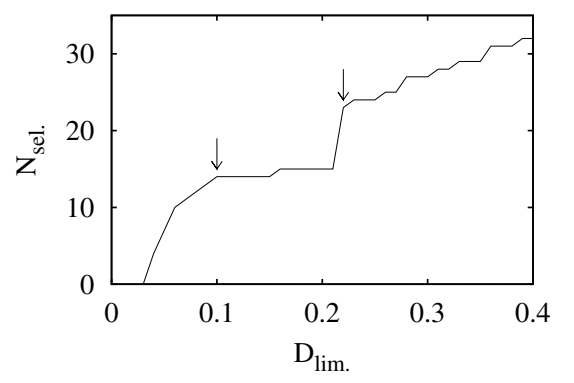

Fig. 6. Dependence of the selected number of December Monocerotids, $N_{\text {sel. }}$, from the photographic IAU MDC database on the limiting value of the D-discriminant. The arrows point the critical values of $\mathrm{D}$ for the selection of the shower's substructure (left) and whole shower (right).

\subsection{April $\rho$-Cygnids}

Filament F1 can be, hypothetically, identified to the April $\rho$-Cygnids (No. 348 in the IAU MDC list), the meteor shower that was recently discovered by the Canadian Meteor Orbit Radar (Brown et al. 2010) and confirmed by the video observations within the Cameras for Allsky Meteor Surveillance (Phillips et al. 2011). The mean radiant of filament F1 is predicted to be situated only $62^{\circ}$ to $65^{\circ}$ from the Sun; therefore, the real meteors of shower No. 348 can be expected to be mainly in the radio data. The particles of filament F1 and, therefore, the real 348 April $\rho$-Cygnids, which originate in C/1917 F1 can approach the Earth's orbit and collide with our planet not earlier than about 20 millennia after their release from the parent-comet nucleus. Despite this relatively long minimum evolutionary period needed, we can find a minor shower that corresponds, in a more or less degree to 348 in the radar data. A "trace" of the shower can also be discerned in the video data (Tables 1 and 2, the part of filament F1). The presence of the shower in the video data is surprising, because the radiant area is situated daily for a much longer time as compared to night sky and one would not expect any significant record of the shower from nighttime observations.

\subsection{Filaments $F 2$ and F4}

These filaments occur only in the models corresponding to the orbital evolution of particles that last 40 and 50 millennia, whereby their prediction is done from a relatively low number of particles (F4 consists of only 2 and 4 particles in the model for 40 and $50 \mathrm{kyr}$, respectively).

Some real meteor associations corresponding to the predicted showers seem to exist in the video and radio data. Their characteristics are given in the parentheses in Tables 1 and 2 in the parts of filaments F2 and F4. We present two sets of characteristics for the association corresponding to filament F2 in the tables, since there is a difference when the iteration to separate the association's members starts from the mean orbit of the shower predicted in the model of $40 \mathrm{kyr}$ and in the model of $50 \mathrm{kyr}$. The associations related to both filaments have not been found earlier; they are present in neither the IAU MDC list of established nor that of working showers.

The presence of the association related to filament $\mathrm{F} 2$ in the video data is especially surprising, since the mean radiant of predicted shower (real association) is only about $24^{\circ}\left(26.5^{\circ}\right)$ from the Sun in the time that corresponds to mean solar longitude of the shower (association).

\section{Discussion}

Some parent bodies may associate a complex of meteor showers, which exhibit, for example, a symmetry of their radiant areas

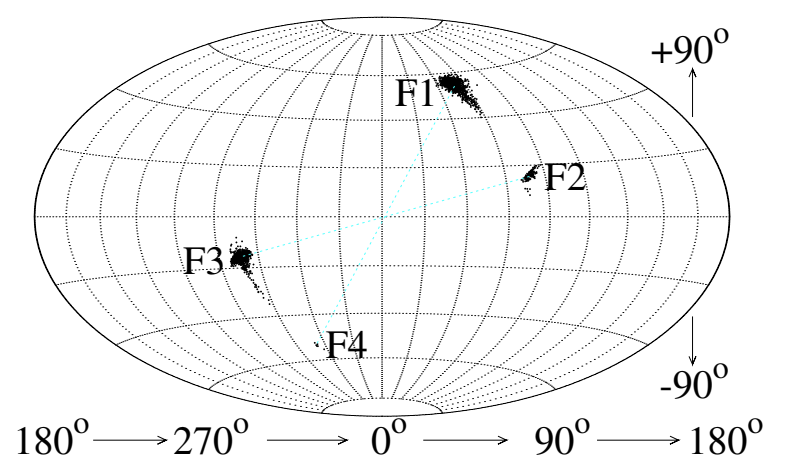

Fig. 7. Positions of the radiants of the particles approaching the Earth's orbit within $0.05 \mathrm{AU}$ at the present in all five models considered. The dashed cyan straight lines connect the mean radiants of filaments F1 and F4 as well as F2 and F3. The ecliptical coordinate frame with the origin in the Earth's apex is used.

with respect to the ecliptic. In the context of cardinal meteor directions, the showers of a complex can be ecliptical, toroidal, or both. An example of the ecliptic-toroidal structure can be found in papers by Neslušan et al. (2013a,b) in the case of almost identical complexes of comet 96P/Machholz and asteroid 2003 EH1.

As seen in Fig. 4, the positions of radiant areas for four filaments of C/1917 F1 also exhibit a certain symmetry. To inspect this symmetry more, we show the positions of the radiants of all theoretical particles in all five models that approach the Earth's orbit within $0.05 \mathrm{AU}$ at the present in the ecliptic coordinate frame in Fig. 7. More specifically, the common ecliptic longitude, $\lambda_{\mathrm{g}}$, is replaced with an angle $\lambda_{\mathrm{A}}=\lambda_{\mathrm{g}}-\left(\lambda_{\odot}+270^{\circ}\right)\left(\lambda_{\odot}\right.$ is the solar longitude corresponding to the time of meteor occurrence) to achieve the identity of the coordinate-frame origin and Earth's apex.

In this projection, we can see that the mean radiant longitude of filaments F1 and F4, which are relatively far from the ecliptic, are different from the longitude of the Earth's apex; therefore, the showers corresponding to these filaments cannot be classified as toroidal. Similarly, filaments F2 and F3, which has radiants situated in a close angular distance to the ecliptic, have no counterpart with a similar ecliptical longitude on the opposite hemisphere; therefore, the corresponding showers are not any regular ecliptical showers.

Nevertheless, there is a clear symmetry for the radiant areas of the pairs, F1-F4 and F2-F3, with respect of the Earth's apex. In Fig. 7, the mean radiants of each pair are connected with dashed, cyan straight lines, and both lines practically cross the origin of the coordinate frame situated in the Earth's apex.

Worthy of discussion is also the large difference in the semimajor axis between the predicted and real showers. In the identification, such a difference is not necessarily a problem. Wu \& Williams (1995) revealed the large dispersion of this orbital element occurring due to the dynamical evolution of the Perseid stream. Later, Kaňuchová et al. (2005) found several discrete filaments of Perseids in the photographic IAU MDC database, whereby the mean semi-major axis of these filaments varied from less than $2 \mathrm{AU}$ up to hyperbolic orbits.

The orbits of particles in the stream of C/1917 F1 do not, however, evolve to the semi-major axes shorter than $\sim 10 \mathrm{AU}$ even in the models of 40 and $50 \mathrm{kyr}$. (The semi-major axis could be more reduced due to non-gravitational effects; Wiegert et al. 2009.) Nevertheless, we know that the eccentricity of the orbit of a real meteor is usually a poorly determined parameter. This is then reflected in a poor determination of the corresponding semi-major axis. Thus, the large discrepancy in the theoretical 
and observed values of semi-major axis is not very significant in the shower identification.

\section{Conclusion remarks}

As discovered earlier (Porter 1952; Hasegawa 1962; McCrosky \& Posen 1961; Lindblad 1971; Kresáková 1974; Drummond 1981; Olson-Steel 1987; Ohtsuka 1989; Lindblad \& OlsonSteel 1990; Vereš et al. 2011), comet C1917 F1 (Mellish) is the parent body of the established meteor shower 19 December Monocerotids. According to our theoretical prediction, this shower should be compact without an internal structure. In the databases of actually observed meteors, we, however, revealed a substructure, which can clearly be discerned inside the main shower. Since this substructure is autonomous, it cannot likely be any result of non-gravitational effects, which act smoothly. The dynamical evolution of some meteoroids of the main DecemberMonocerotid stream may also not lead to its occurrence because such an effect would have to be revealed in our simulations. We can speculate that the nucleus of C/1917 F1 partially split in the past, and a fragment separated from it. This fragment then became the parent body of the substructure.

Possibly, comet C/1917 F1 also associates 348 April $\rho$-Cygnids. In the models, the corresponding filament F1 is numerous and, hence, this shower is well predicted. The similarity of the mean characteristics of the predicted and real showers is, however, only vague. It does not enable us to draw a clear conclusion.

In the theoretical long-period models, the symmetrical filaments with respect to the Earth's apex occur. Unfortunately, no real counterparts of these symmetrical parts can be surely identified in the databases. The clear absence of the real symmetrical parts is probably caused by the relatively long period needed for the stream to evolve into the corresponding filaments. The Earth's apex symmetrical counterpart of 19 December Monocerotids would be, if existed, a typical daytime shower.

In the databases, 250 November Orionids can be found in the orbital phase space that is similar to that of 19 December Monocerotids. However, shower No. 250 is obviously not directly related to $\mathrm{C} / 1917 \mathrm{~F} 1$, since no single theoretical particle in all five models is in an orbit similar to the mean orbit of this shower. Real 250 November Orionids appear to be even less numerous (seven meteors in the IAU MDC photographic database and 92 meteors in the SonotaCo video database belong to the dense part of the shower; the radio data were not scanned) than the above mentioned substructure of December Monocerotids.

The November Orionids are well separated from the December Monocerotids and, hence, it is improbable that some non-gravitational effects, which we did not consider in our work, could change the orbits of its members in such a way that the separation would occur. We can, however, again speculate that this low-numerous shower originates from a fragment that escaped from the C/1917 F1 nucleus. It likely happened a relatively long time ago; therefore, the fragment had enough time to come in an orbit, which makes it less similar to the orbit of the parent comet than that of the parent fragment of the DecemberMonocerotid substructure, or, there exists another parent body of the November Orionids, which is not related to C/1917 F1 and will be possibly discovered in future.

Acknowledgements. This article was supported by the realization of the Project ITMS No. 26220120009, based on the supporting operational Research and development program financed from the European Regional Development Fund. The work was also supported, in part, by the VEGA - the Slovak Grant Agency for Science, grant No. 2/0031/14 and by the Slovak Research and Development Agency under the contract No. APVV-0517-12.

\section{References}

Asher, D. J. 1999, MNRAS, 307, 919

Asher, D. J. 2005, in Dynamics of Populations of Planetary Systems, eds. Z. Knežević, \& A. Milani (Cambridge: Cambridge Univ. Press), Proc. IAU Coll., 197, 375

Asher, D. J. 2008, Earth Moon Planets, 102, 27

Asher, D. J., \& Emel'yanenko, V. V. 2002, MNRAS, 331, 126

Asher, D. J., Bailey, M. E., \& Emel'yanenko, V. V. 1999a, Irish Astron. J., 26, 91

Asher, D. J., Bailey, M. E., \& Emel'yanenko, V. V. 1999b, MNRAS, 304, L53

Asklöf, S. 1932, Arkiv for Mat. Astron. och Fys., 23A, 11

Babadzhanov, P. B., Williams, I. P., \& Kokhirova, G. I. 2008, MNRAS, 386, 2271

Babadzhanov, P. B., Williams, I. P., \& Kokhirova, G. I. 2013, A\&A, 556, A25

Beech, M. 2001, MNRAS, 327, 1201

Brown, P., \& Jones, J. 1998, Icarus, 133, 36

Brown, P., Wong, D., Weryk, R., \& Wiegert, P. 2010, Icarus, 207, 66

Chambers, J. E. 1999, MNRAS, 304, 793

Drummond, J. 1981, Icarus, 45, 545

Everhart, E. 1985, in Dynamics of Comets: Their Origin and Evolution, eds. A. Carusi, \& G. B. Valsecchi (Dordrecht: Kluwer), 185

Hasegawa, I. 1962, in General Index List of Theoretical Radiant Points of Meteors Associated with Comets, 3rd edn. (Paris: Doc. Des Observateurs), 14

Hawkins, G. S. 1963, Smithsonian Contr. Astrophys., 7, 53

Hindley, K. B., \& Houlden, M. A. 1970, Nature, 225, 1232

Jenniskens, P. 2006, Meteor Showers and Their Parent Comets (Cambridge: Cambridge Univ. Press)

Jenniskens, P., \& Vaubaillon, J. 2010, AJ, 139, 1822

Jopek, T. J., \& Williams, I. P. 2013, MNRAS, 430, 2377

Kaňuchová, Z., Svoreň, J., \& Neslušan, L. 2005, Contrib. Astron. Obs. Skalnaté Pleso, 35, 135

Kresáková, M. 1974, Bull. Astron. Inst. Czechosl., 9, 88

Lindblad, B. A. 1971, Space Research, 11, 287

Lindblad, B. A., \& Olsson-Steel, D. 1990, Bull. Astron. Inst. Czechosl., 41, 193

Lindblad, B. A., Neslušan, L., Porubčan, V., \& Svoreň, J. 2003, Earth, Moon, Planets, 93, 249

Lyytinen, E., \& Jenniskens, P. 2003, Icarus, 162, 443

McCrosky, R. E., \& Posen, A. 1961, Smithson. Contr. Astrophys., 4, 15

Neslušan, L. 1999, A\&A, 351, 752

Neslušan, L., Svoreň, J., \& Porubčan, V. 1995, Earth, Moon, Planets, 68, 427

Neslušan, L., Kaňuchová, Z., \& Tomko, D. 2013a, A\&A, 551, A87

Neslušan, L., Hajduková, M., jr., \& Jakubík, M. 2013b, A\&A, 560, A47

Neslušan, L., Svoreň, J., \& Porubčan, V. 2013c, Earth Moon Planets, 110, 41

Ohtsuka, K. 1989, WGN, 17, 93

Olsson-Steel, D. 1987, Austral. J. Astron., 2, 21

Phillips, M., Jenniskens, P., Grigsby, B. 2011, WGN - Journal of the IMO, 39, 131

Porter, J. K. 1952, in Comets and Meteor Streams, Chapman and Hall, London, 123

Sekanina, Z., \& Southworth, R. B. 1975, Physical and dynamical studies of meteors, Meteor-fragmentation and stream-distribution studies, Final Report (Cambridge, MA: Smithsonian Astrophys. Obs.)

Sekhar, A., \& Asher, D. J. 2013, MNRAS, 433, 84

Sekhar, A., \& Asher, D. J. 2014a, Meteor. Planet. Sci., 49, 52

Sekhar, A., \& Asher, D. J. 2014b, MNRAS, 437, L71

SonotaCo. 2009, WGN - Journal of the IMO, 37, 55 [http://sonotaco.jp/ $\mathrm{doc} / \mathrm{SNM} /]$

Tomko, D. 2014, Contrib. Astron. Obs. Skalnaté Pleso, 44, 33

Tomko, D. 2013, Ph.D. dissertation (in Slovak), Tatranská Lomnica: Faculty of Mathematics, Physics, and Informatics of the Commenius Univ. and Astronomical Inst. of the Slovak Acad. Sci., Slovakia

Tomko, D., \& Neslušan, L. 2012, Earth, Moon, Planets, 108, 123

Vaubaillon, J., \& Jenniskens, P. 2007, Adv. Space Res., 39, 612

Vaubaillon, J., Arlt, R., Shanov, S., Dubrovski, S., \& Sato, M. 2005a, MNRAS, 362,1463

Vaubaillon, J., Colas, F., \& Jorda, L. 2005b, A\&A, 439, 751

Vereš, P., Kornoš, L., \& Tóth, J. 2011, MNRAS, 412, 511

Whipple, F. L. 1954, AJ, 59, 201

Wiegert, P. A., Brown, P. G., Vaubaillon, J., \& Schijns, H. 2005, MNRAS, 361, 638

Wiegert, P., Vaubaillon, J., \& Campbell-Brown, M. 2009, Icarus, 201, 295

Williams, I. P., \& Wu, Z. 1994, MNRAS, 269, 524

Wu, Z., \& Williams, I. P. 1995, MNRAS, 276, 1017 